\title{
SHEAVES OF $H$-SPACES AND SHEAF COHOMOLOGY
}

\author{
BY \\ JAMES M. PARKS $\left({ }^{1}\right)$
}

\begin{abstract}
The concept of a sheaf of $H$-spaces is introduced and, using the Cech technique, a cohomology theory is defined in which the cohomology "groups" are $H$-spaces. The corresponding axioms of Cartan [3] for this theory are verified and other properties of the theory are investigated.
\end{abstract}

0. Introduction. In defining sheaves of algebraic structures the local homeomorphism condition is central in order to topologize the sheaf space in such a way as to preserve the (discrete) stalkwise structures. If the stalks are allowed to support a nondiscrete structure (such as an $H$-structure) then the local homeomorphism condition will no longer suffice. However by weakening this local condition (such that the local path-connectedness is preserved under the projection (open) map) a topology may be introduced in the sheaf space in such a way as to preserve the stalk structures and include the discrete situation as a special case. This is the basic aim of this paper.

In $\S 1$ the definition of a sheaf of $H$-spaces is introduced and examples and terminology are covered. Particular attention should be paid to the main example below as a motivation for such sheaves vs. sheaves of algebraic structures. The cohomology theory is defined in $\S 2$ using the Cech construction [2, pp. 470471], and the axioms for this theory (cf. [3]) are demonstrated in $\$ 3$. In $\S 4$ the excision property is covered, and in $\S 5$ the homotopy property for this cohomology is proved.

All base and stalk spaces are assumed to be Hausdorff and the term mapping always means continuous mapping. Finite indexes are denoted by $\pi$. The term $H$-space means a space with a continuous multiplication (an $H$-structure) which admits a two-sided identity (up-to-homotopy). (It is not assumed the stalk spaces satisfy the homotopy extension property.) Terminology will follow [10] with particular attention to Definition 1.3 whenever maps of sheaves of $H$-spaces are involved.

\footnotetext{
Received by the editors March 5, 1973 and, in revised form, March 25, 1974. $18 \mathrm{~F} 20$.

AMS (MOS) subject classifications (1970). Primary 55B30, 55D45; Secondary 55B05,

Key words and phrases. Sheaves, $H$-spaces, sheaf cohomology, Cech theory, sheaf cohomology axioms.

(1) The author was partially supported by NDEA TITLE IV FELLOWSHIPS while a doctoral student at the University of Houston during the preparation of parts of this work. 
The author wishes to thank his advisor Professor D. G. Bourgin for several helpful conversations and the referee for his valuable suggestions during the preparation of this work.

\section{Sheaves of $H$-spaces.}

Definition 1.1. If $\left\{H_{x}, \mu_{x}\right\}_{X}$ is a collection of $H$-spaces (and corresponding multiplications) indexed by a given space $X$, let $H=\bigcup_{X} H_{x}$ and let the function $p: H \rightarrow X$ be defined by $p\left(H_{x}\right)=x$. A basis for a topology on $H$ is determined as follows. Given a point $a$ in $H$, a set $N$ in $H$ which contains $a$ is called open iff $p(N)$ is open in $X$ and $N \cap H_{x}$ is open and path connected in $H_{x}$ for all $x$ in $X$. If for each point in $H$ a path-connected neighborhood in the basis determined above exists and the operations $\mu_{x}$ are continuous in this topology on $H$, then the structure $(H, p, X)$ (or $H$ when $X$ is understood) is called a sheaf of $H$ spaces. Clearly a sheaf of algebraic structures is a sheaf of $H$-spaces.

EXAMPLES. Let $p: E \rightarrow B$ be a map such that $p^{-1}(x) \simeq F, F$ a given space (a quasi-fibration [8], [10] for instance), and let $E^{\prime}=\left\{\varphi \in E^{F} \mid \varphi: F \cong p^{-1}(x)\right.$ for some $x \in B$ \}. Then if $p^{\prime}: E^{\prime} \rightarrow B$ is defined by $p^{\prime}(\varphi)=p(\varphi(F))$, one has $p^{\prime-1}(x)=F^{\prime}=\{\varphi: F \rightarrow F \mid \varphi$ is a homotopyequivalence $\}$, i.e. $p^{\prime}$ is the associated (principal) map to $p$ [9]. If $E$ reflects the local path-connectedness of $B$ via $p$ as in 1.1 above then the space $E^{\prime}$ is a sheaf of $H$-spaces (of type $F^{\prime}$ ) over $B$.

An example of a sheaf of $H$-spaces which is not itself an $H$-space is the sheaf $\left(S^{2}, p, D\right), D$ the unit disc in the plane, with $p$ the projection of $S^{2}$ onto $D$. (The only spheres which support $H$-structures are $S^{0}, S^{1}, S^{3}$, and $S^{7}$ [1].)

Definition 1.2. Let $\theta: X \rightarrow H$ denote a (not necessarily unique) section which satisfies: $\theta(x) \in C\left(e_{x}\right) \subset H_{x}$ for all $x$ in $X$, where $C\left(e_{x}\right)$ is the component of the identity in $H_{x}$.

Let $S(X, H)$ denote the collection of global sections of $H$ with the compactopen topology. Define a multiplication $\mu$ on $S(X, H)$ as follows: if $s, t \in S(X, H)$, then for each $x \in X$

$$
\mu(s, t)(x)=\mu_{x}(s(x), t(x)) .
$$

Continuity is immediate. Note $\mu$ is continuous if $X$ is locally compact, however the following argument demonstrates this condition is not necessary. Let $U$ be an open set about $\mu(s, t)$ in $S(X, H)$. Then there exists a finite collection of open sets in the subbasis, $\left\{M\left(C_{i}, O_{i}\right)\right\}_{i \in \pi}$, such that $\mu(s, t) \in \bigcap_{i \in \pi} M\left(C_{i}, O_{i}\right) \subset U$. Let $U_{i}$ be a neighborhood of $s(x)$ in $H$, and $V_{i}$ a neighborhood of $t(x)$ in $H, x \in$ $C_{i}$, such that if $s_{i}$ and $t_{i} \in S(X, H)$ such that $s_{i}\left(x^{\prime}\right) \in U_{i}$ and $t_{i}\left(x^{\prime}\right) \in V_{i}$, where $x^{\prime} \in C_{i}$, then $\mu\left(s_{i}, t_{i}\right)\left(x^{\prime}\right)=\mu_{x^{\prime}}\left(s_{i}\left(x^{\prime}\right), t_{i}\left(x^{\prime}\right)\right) \in O_{i}$. Thus $s \in \bigcap M\left(C_{i}, U_{i}\right)$ and

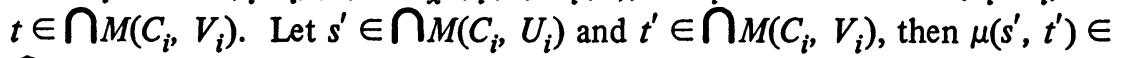
$\bigcap M\left(C_{i}, O_{i}\right)$ since $\mu\left(s^{\prime}, t^{\prime}\right)(x)=\mu_{x}\left(s^{\prime}(x), t^{\prime}(x)\right) \in O_{i}$ whenever $x \in C_{i}$ by definition 
of $U_{i}$ and $V_{i}$. An identity (up-to-homotopy) for $S(X, H)$ is the section $\theta$. The notation $s \circ t$ will be used for $\mu(s, t)$ below.

If $U \subset X$, then $S(U, H \mid U)$, where $H \mid U=p^{-1}(U)$, is an $H$-space under the induced multiplication from $S(X, H)$. Denote $S(U, H \mid U)$ by $S(U, H)$ below.

Let $F$ be a family of supports on $X$ and $U \subset X$. Then $S_{F \mid U}(U, H)$ is the collection of sections $s \in S(U, H)$ which satisfy $|s| \in F \mid U$, where $F \mid U=\{A \subset U \mid$ $A \in F\}$ and $|s|=\left\{x \in X \mid s(x) \notin C\left(e_{x}\right)\right\}$. The collection $S_{F \mid U}(U, H)$ is closed under the multiplication of $S(X, H)$ restricted to $S_{F \mid U}(U, H)$, for if $s, t \in$ $S_{F \mid U}(U, H)$, then $|s|,|t| \in F \mid U$ and, since $\left|\mu_{F \mid U}(s, t)\right|^{\sim} \supset|s| \sim|t| \sim$, it follows that $\left|\mu_{F \mid U}(s, t)\right| \subset|s| \cap|t|$ and thus $\left|\mu_{F \mid U}(s, t)\right| \in F \mid U$, or $S_{F \mid U}(U, H)$ is an $H$ space.

Definition 1.3. Let $H$ and $K$ be sheaves of $H$-spaces on $X$. A map of sheaves of $H$-spaces $\alpha: H \rightarrow K$ is a map such that

$$
\begin{gathered}
p_{2} \alpha=p_{1}, \\
\alpha_{x}: H_{x} \rightarrow K_{x} \text { is an } H \text {-map [10]. }
\end{gathered}
$$

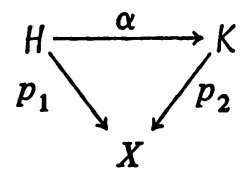

Note, if $\alpha$ is a map of sheaves of $H$-spaces $H \rightarrow K$, then $H \approx K$ iff $\alpha_{x}: H_{x} \rightarrow K_{x}$ is a homotopy equivalence for all $x \in X$.

The map $\alpha$ induces a map $\alpha^{\prime}: S(X, H) \rightarrow S(X, K)$ by the rule $\alpha^{\prime}(s)=\alpha s$, $s \in S(X, H)$. The section functor $S$ is thus a functor on the category of sheaves of $H$-spaces on some fixed base space to the category of $H$-spaces and $H$-maps.

DEFINITION 1.4. Let $V \subset U \subset X$ be open sets and define as usual the map

$$
r_{V}^{U}: S(U, H) \rightarrow S(V, H)
$$

by restriction. This map is clearly an $H$-map, and the collection

$$
\Sigma=\left\{S(U, H), r_{V}^{U}\right\}
$$

forms a direct system.

DEfinition 1.5. A presheaf $P$ of $H$-spaces on $X$ is a contravariant functor on $T_{X}$ and inclusions to the category of $H$-spaces and restrictions ( $H$-word-maps).

Let $M=\bigcup_{U \in T_{X}}(U \times P(U))$ and define $(x, a) \sim(y, b)$ iff $x=y$ and there exists a neighborhood of $x$, say $W \subset U \cap V$, such that $P\left(i_{W}{ }^{U}\right)(\mathbf{a})=P\left(i_{W}{ }^{V}\right)(\mathbf{b})$, where $(x, \mathrm{a}) \in U \times P(U)$ and $(y, \mathrm{~b}) \in V \times P(V)$ and $i_{W}{ }^{U}$ is inclusion. 
Form the quotient space $\widetilde{H}=M / \sim$ (the quotient topology is assumed). Let $\pi: \widetilde{H} \rightarrow X$ be the projection map induced by $p: M \rightarrow X$, where $p(x, a)=x$ (note $\pi$ is open since $p$ is open and $\tilde{p}$ is continuous, where $\tilde{p}: M \rightarrow \widetilde{H}$ is the natural map).

Consider $\pi^{-1}(x)=\widetilde{H}_{x}=\{\langle x, a)\}$. This is clearly the limit:

$$
\underset{x \in U}{\mathbf{L}}\left\{P(U), r^{U}{ }_{w}\right\}=\left\{\langle\mathbf{a}\rangle \mid(y, b) \in\langle\mathrm{a}\rangle \text { iff } r^{U}{ }_{w}(\mathbf{a})=r^{V}{ }_{w}(\mathbf{b}), x \in W \subset U \cap V\right\},
$$

and has a natural $H$-structure. Thus $\tilde{H}$ is essentially $\bigcup_{x \in X} \widetilde{H}_{x}$.

Since the stalkwise multiplications in $M$ are continuous, they are so in $\widetilde{H}$, and $\tilde{H}$ is called the sheaf of $H$-spaces generated by $P$ on $X$.

Note if $H$ is a sheaf of $H$-spaces and $P$ is the presheaf of sections of $H$, there is a map $\psi: H \rightarrow \widetilde{H}$ defined by $\psi\left(a_{x}\right)=\langle a\rangle_{x}=\{s \mid s(x) \simeq a\}$ which preserves the $H$-structure and is a homotopy equivalence stalkwise, with inverse $\langle a\rangle_{x}=a_{x}$, where $s^{\prime}(x) \simeq \mathrm{a}$ for all $s^{\prime} \in\langle\mathrm{a}\rangle_{x}$. Thus $H \approx \widetilde{H}$ as sheaves of $H$-spaces by 1.3 .

2. Sheaf cohomology. It is assumed below that the stalk $H$-structures are homotopy-associative, homotopy-commutative and admit homotopy inverses [10] which are continuous in the topology of the sheaf space. These restrictions are sufficient for this construction. The $H$-space $S(U, H)$ inherits these additional properties through the induced $H$-structure. A sheaf cohomology with coefficients in a sheaf of $H$-spaces will be defined using the Cech technique (cf. [2], [4]).

Definition 2.1. Let $\omega=\left\{w_{i}\right\}$ and $\nu=\left\{v_{j}\right\}$ be open covers of $X$ with corresponding nerves $w$ and $v$. If the nucleus $N=\bigcap_{m=0}^{q} w_{i_{m}} \neq \varnothing$ then $w_{i_{0}} \ldots w_{i_{q}}$ is a $q$-simplex (which is denoted by $i_{0} \ldots i_{q}$ for convenience). Let $w(q)$ denote the collection of $q$-simplexes in $w$.

Define the $q$-cochains of $w$ with coefficients in $\Sigma$ (see (1.4b)) by

$$
C^{q}(w, \Sigma)=\left\{f^{q}: w(q) \rightarrow \Sigma \mid f^{q}\left(i_{0} \ldots i_{q}\right) \in S(N, H)\right\},
$$

$N$ the nucleus of $i_{0} \ldots i_{q}$. Topologize $C^{q}(w, \Sigma)$ with the compact-open topology.

Define a multiplication on $C^{q}(w, \Sigma)$ by the rule:

$$
\left(f^{q} \circ g^{q}\right)\left(i_{0} \ldots i_{q}\right)=\mu_{N}\left(f^{q}\left(i_{0} \ldots i_{q}\right), g^{q}\left(i_{0} \ldots i_{q}\right)\right),
$$

where $f^{q}, g^{q} \in C^{q}(w, \Sigma)$.

If $\theta^{q}$ denotes a map $i_{0} \ldots i_{q} \rightarrow \theta_{N} \in S(N, H)$, then by an argument similar to that of $1.2 C^{q}(w, \Sigma)$ is an $H$-space with a homotopy-associative and homotopycommutative $H$-structure which admits homotopy-inverses.

Let

$$
C_{F}^{q}(w, \Sigma)=\left\{f^{q} \in C^{q}(w, \Sigma)|| f^{q} \mid \in F\right\},
$$


where $\left|f^{q}\right|$ is the closure of the union $\bigcup\left|f^{q}\left(i_{0} \ldots i_{q}\right)\right|$ taken over $w(q)$. By 1.2 and the above definition of multiplication on $C^{q}(w, \Sigma)$ the space $C_{F}^{q}(w, \Sigma)$ is an $H$-space under the induced multiplication of $C^{q}(w, \Sigma)$.

Definition 2.2. Define a map $d: C_{F}^{q}(w, \Sigma) \rightarrow C_{F}^{q+1}(w, \Sigma)$ by

$$
\left(d f^{q}\right)\left(i_{0} \ldots i_{q+1}\right)=\bigcirc_{k=0}^{q+1}(\iota) r^{N_{k}} f^{q}\left(i_{0} \ldots \hat{k} \ldots i_{q+1}\right),
$$

where $N=\bigcap_{m=0}^{q+1} w_{i_{m}} \neq \varnothing ; N_{k}=\bigcap_{m \neq k} w_{i_{m}} \neq \varnothing$, for $k=0, \ldots, q+1$; $\iota$ is the homotopy-inverse on $S(N, H)$ and $(\iota)$ denotes $\iota$ on the odd terms and the identity (up-to-homotopy) on the even terms. The right-hand side of equation (2.2a) is a shorthand notation for the product:

$$
r^{N_{0}}{ }_{N} f^{q}\left(i_{1} \ldots i_{q+1}\right) \circ \iota r^{N_{1}} f^{q}\left(i_{0} i_{2} \ldots i_{q+1}\right) \circ \ldots
$$

$$
\circ(\iota) r^{N_{k}}{ }_{N} f^{q}\left(i_{0} \ldots \hat{k} \cdots i_{q+1}\right) \circ \ldots \circ(\iota) r^{N} q+1{ }_{N} f^{q}\left(i_{0} \ldots i_{q}\right) .
$$

LEMmA 2.3. The map $d$ is an H-map and $d^{2}$ is trivial (i.e. $d^{2} \simeq \theta$ ).

Proof. Let $f^{q}, g^{q} \in C_{F}^{q}(w, \Sigma)$ and $\left(i_{0} \ldots i_{q+1}\right) \in w(q+1)$. Then

(2.3a) $\left.\left(d\left(f^{q} \circ g^{q}\right)\right)\left(i_{0} \ldots i_{q+1}\right) \simeq \underset{k=0}{q+1}(\iota) r^{N_{k}} f^{q} \circ r^{N_{k}}{ }_{N} g^{q}\right)\left(i_{0} \ldots \hat{k} \cdots i_{q+1}\right)$, and

$$
\left(d f^{q} \circ d g^{q}\right)\left(i_{0} \ldots i_{q+1}\right)=\left(\underset{k=0}{q+1}(\iota) r{ }^{N_{k}} f^{q}\left(i_{0} \ldots \hat{k} \ldots i_{q+1}\right)\right)
$$

$$
\circ\left(\underset{k=0}{q+1}(\iota) r^{N}{ }_{N} g^{q}\left(i_{0} \cdots \hat{k} \cdots i_{q+1}\right)\right) .
$$

Since the $H$-structures are homotopy-commutative, (2.3a) and (2.3b) are homotopic and $d$ is an $H$-map.

Let $\left(i_{0} \ldots i_{q+2}\right) \in w(q+2)$, then

$$
\begin{aligned}
& \left(d^{2} f^{q}\right)\left(i_{0} \ldots i_{q+2}\right) \\
& =\bigcirc_{k=0}^{q+2}(\iota) r^{N}{ }_{N}\left(\underset{j=0 ; j \neq k}{\bigcirc^{q+2}}(l) r^{N}{ }_{k j}{ }_{k} f^{q}\left(i_{0} \cdots \hat{j} \cdots \hat{k} \cdots i_{q+2}\right)\right),
\end{aligned}
$$

where $N_{k j}=\bigcap_{m \neq j ; m \neq k} w_{i_{m}}$. 
By definition of the restriction maps (1.4a) $r^{N}{ }^{k}{ }{ }^{N}{ }^{N j}{ }_{N_{k}} \simeq r^{N}{ }_{k j}$. (Note also $N_{k j}=N_{j k}$ and ८०८ $\simeq 1$.) By expanding (2.3c) and applying this information one has

$$
\left(d^{2} f^{q}\right)\left(i_{0} \ldots i_{q+2}\right) \cong \theta^{q+2}\left(i_{0} \ldots i_{q+2}\right)=\theta_{N} \in S(N, H) .
$$

DEFINITION 2.4. Let $\operatorname{Ker} d^{q}=\left\{f^{q} \mid d f^{q} \cong \theta^{q+1}\right\}$ and $\operatorname{Im} d^{q}=$ $\left\{f^{q+1} ! f^{q+1} \simeq d^{q} f^{q}\right.$ for some $\left.f^{q}\right\}$.

Lemma 2.5. $\operatorname{Im} d^{q}$ and $\operatorname{Ker} d^{q}$ are H-spaces for $q \geqslant 0$.

Proof. If $f^{q}, g^{q} \in \operatorname{Ker} d^{q}$, then clearly $f^{q} \circ g^{q} \in \operatorname{Ker} d^{q}$, and $\operatorname{Ker} d^{q}$ is an $H$-space under the multiplication of $C_{F}^{q}(w, \Sigma)$.

Let $f^{q+1}, g^{q+1} \in \operatorname{Im} d^{q}$. Then there exist elements $f^{q}, g^{q} \in C_{F}^{q}(w, \Sigma)$ such that $f^{q+1} \simeq d^{q} f^{q}$ and $g^{q+1} \simeq d^{q} g^{q}$. Thus $f^{q+1} \circ g^{q+1} \in \operatorname{Im} d^{q}$ (also $\theta^{q+1} \in \operatorname{Im} d^{q}$, since $d^{q} \theta^{q} \simeq \theta^{q+1}$ ), and $\operatorname{Im} d^{q}$ is an $H$-space.

Definition 2.6. By $2.3 \operatorname{Im} d^{q-1} \subset \operatorname{Ker} d^{q}$ for $q \geqslant 1$. Define

$$
H_{F}^{q}(w, \Sigma)=\left\{f^{q} \circ \operatorname{Im} d^{q-1} \mid f^{q} \in \operatorname{Ker} d^{q}\right\},
$$

where $f^{q} \circ \operatorname{Im} d^{q-1}=\left\{f^{q} \circ g^{q} \mid g^{q} \in \operatorname{Im} d^{q-1}\right\}$ (recall the definition of $\operatorname{Im} d^{q-1}$ above (2.4)). Recall also (2.1) the $H$-structures involved are homotopy-commutative, thus $H_{F}^{q}(w, \Sigma)$ is well defined (as $\operatorname{Im} d^{q-1}$ behaves as a normal subgroup in view of 2.4, i.e. $\operatorname{Im} d^{q-1}$ determines an equivalence relation on $\operatorname{Ker} d^{q}$ which is natural with respect to the $H$-structure on $\left.\operatorname{Ker} d^{q} 2.5\right)$. Under the quotient topology an $H$-structure is induced on $H_{F}^{q}(w, \Sigma)$ by the rule

$$
\mu^{\prime}\left(f^{q} \circ \operatorname{Im} d^{q-1}, g^{q} \circ \operatorname{Im} d^{q-1}\right)=\left(f^{q} \circ g^{q}\right) \circ \operatorname{Im} d^{q-1} .
$$

Let $\left\langle f^{q}\right\rangle$ denote the coset $f^{q} \circ \operatorname{Im} d^{q-1}$ and $\theta$ denote $\theta^{q} \circ \operatorname{Im} d^{q-1}$ below.

The induced map $\mu^{\prime}$ is continuous by the following argument. Let $\left\langle f^{q}\right\rangle$, $\left\langle g^{q}\right\rangle \in H_{F}^{q}(w, \Sigma)$ and let $h^{\prime}=\mu^{\prime}\left(\left\langle f^{q}\right\rangle,\left\langle g^{q}\right\rangle\right)$. Let $U$ be a neighborhood of $h^{\prime}$, then by the quotient topology $U=\left\{\left\langle j^{q}\right\rangle\right\}$, where $j^{q} \in V \subset \operatorname{Ker} d^{q}, V$ open. Now $h^{\prime} \in U$ so $h^{\prime}=h^{q} \circ \operatorname{Im} d^{q-1}$ for some $h^{q} \in V$. By the continuity of $\mu$ there exist neighborhoods $W_{1}$ and $W_{2}$ of $f^{q}$ and $g^{q}$, respectively, such that $\mu\left(m^{q}, n^{q}\right) \in V$ whenever $m^{q} \in W_{1}$ and $n^{q} \in W_{2}$. Thus if $W_{1}^{\prime}$ is the open set determined by $W_{1}$ and $W_{2}^{\prime}$ is the open set determined by $W_{2}$ (in $H_{F}^{q}(w, \Sigma)$ ), then $\mu^{\prime}\left(\left\langle m^{q}\right\rangle,\left\langle n^{q}\right\rangle\right) \in U$ whenever $\left\langle m^{q}\right\rangle \in W_{1}^{\prime}$ and $\left\langle n^{q}\right\rangle \in W_{2}^{\prime}$.

Definition 2.7. If $\nu$ and $\omega$ are open covers of $X$ and $\omega$ refines $\nu, \nu<\omega$, let $p^{w}{ }_{v}$ denote a (nonunique) projection map of simplexes $\left(i_{0} \ldots i_{q}\right) \in w$ into simplexes $\left(j_{0} \ldots j_{p}\right) \in v, p \leqslant q$.

This map induces a map $p_{v}^{w \#}: C_{F}^{q}(v, \Sigma) \rightarrow C_{F}^{q}(w, \Sigma)$ by the rule: 


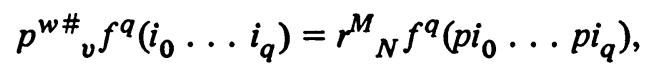

where $N=\bigcap w_{i_{m}}, M=\bigcap v_{j_{k}=p i_{m}}$, and $f^{q} \in C_{F}^{q}(v, \Sigma)$.

Note $\left|p^{w \#}{ }_{v} f^{q}\right| \subset\left|f^{q}\right|$, and $p^{w \#}{ }_{v}$ is an $H$-map since $r^{M}$ is an $H$-map (1.4).

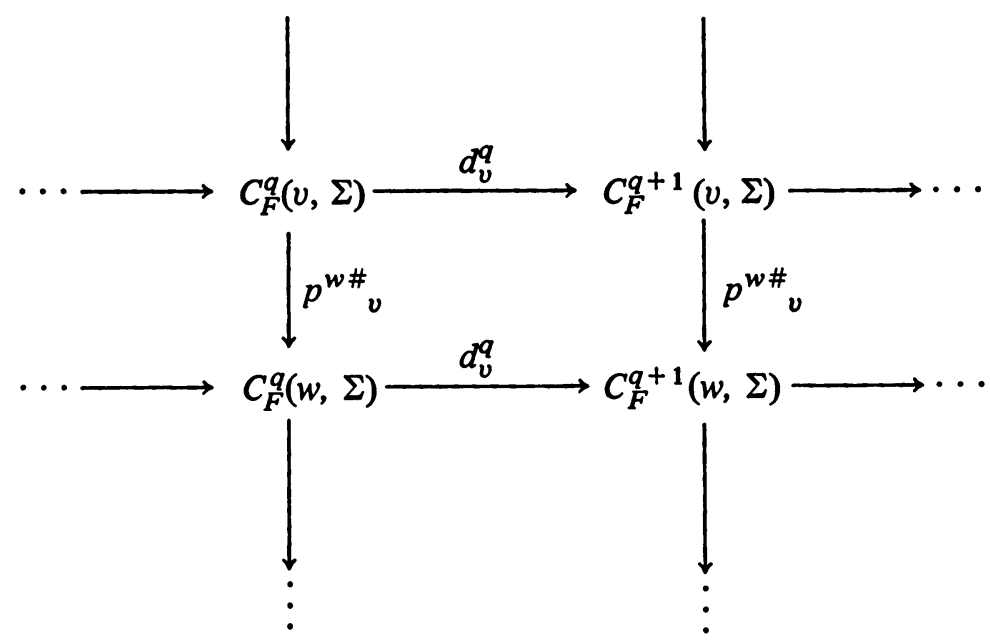

LEMma 2.8. If $\nu<\omega$ then for $q \geqslant 1$

$$
\begin{gathered}
d_{w}^{q} p^{w \#} \simeq p_{v}^{w \#} d_{v}^{q}, \\
p^{w \#}\left(\operatorname{Ker} d_{v}^{q}\right) \subset \operatorname{Ker} d_{w}^{q}, \\
p^{w \#}{ }_{v}\left(\operatorname{Im} d_{v}^{q-1}\right) \subset \operatorname{Im} d_{w}^{q-1} .
\end{gathered}
$$

Proof. (2.8a) is immediate, (2.8b) follows from (2.8a), and (2.8c) follows by a standard diagram chasing argument (see diagram above).

DEFINITION 2.9. By (2.8) $p^{w \#}$ induces an $H$-map $p^{w *}: H_{F}^{q}(v, \Sigma) \rightarrow$ $H_{F}^{q}(w, \Sigma)$ by the rule:

$$
p^{w *}\left(\left\langle f^{q}\right\rangle\right)=\left\langle p^{w \#} f_{v}^{q}\right\rangle
$$

where $\left\langle f^{q}\right\rangle \in H_{F}^{q}(v, \Sigma)$.

The uniqueness of the induced map $p^{w *}$ is demonstrated by the following lemma. $p^{\prime w *}$

LEMMA 2.10. If $p^{w}{ }_{v}$ and $p^{\prime w}$ are projection maps of $w$ to $v$, then $p^{w *}{ }_{v}=$ Proof. Define as usual a map $D: C_{F}^{q}(w, \Sigma) \rightarrow C_{F}^{q-1}(w, \Sigma)$ by 


$$
D f^{q}\left(i_{0} \ldots i_{q-1}\right)=\underset{k=0}{q-1}(\iota) r^{M}{ }_{N} f^{q}\left(p i_{0} \ldots p i_{k} p^{\prime} i_{k} \ldots p^{\prime} i_{q-1}\right),
$$

where $M_{k}=\left(\bigcap_{m=0}^{k} w_{p i_{m}}\right) \cap\left(\bigcap_{m=k}^{q-1} w_{p^{\prime} i_{m}}\right)$, and $N=\bigcap_{v_{i}}$. Then

$$
\left(D d f^{q} \circ d D f^{q}\right)\left(i_{0} \ldots i_{q}\right) \simeq\left(p^{\prime w \#}{ }_{v}^{\circ} p^{w \#}{ }_{v}\right)\left(i_{0} \ldots i_{q}\right) .
$$

If $\left\langle f^{q}\right\rangle \in H_{F}^{q}(v, \Sigma)$, then $f^{q} \in \operatorname{Ker} d^{q}$ and $D d f^{q}=\theta^{q}$, while $d D f^{q} \in$ $\operatorname{Im} d^{q-1}$. Thus $\left\langle(D d \circ d D) f^{q}\right\rangle=\theta$ and $p^{\prime w *}{ }_{v}=p^{w *}{ }_{v}$.

DEFINITION 2.11. By 1.4 and 2.7 the collection $\left\{H_{F}^{*}(w, \Sigma), p^{w *}{ }_{v}\right\}$ forms a homotopy-direct system. $\left({ }^{2}\right)$ Define the cohomology of $X$ with values in $H$ and supports in $F$ as the homotopy-direct-limit $\left({ }^{3}\right)$ of this system

$$
H_{F}^{p}(X, H)=\underset{w}{L}\left\{H_{F}^{p}(w, \Sigma), p^{w *}\right\} .
$$

THEOREM 2.12. $H_{F}^{*}(X, H)$ is an H-space.

Proof. If $\alpha<\beta<\nu<\omega$, then

$$
p^{w *}\left(\mu_{v}\left(p^{v *}{ }_{a}\left(\langle x\rangle_{a}\right), p^{v *}{ }_{b}\left(\langle x\rangle_{b}\right)\right)\right) \simeq \mu_{w}\left(p_{a}^{w *}\left(\langle x\rangle_{a}\right), p^{w *}{ }_{b}\left(\langle x\rangle_{b}\right)\right),
$$

and

$$
\mu_{v}\left(p_{a}^{b *}\left(\langle x\rangle_{a}\right), p^{b *}{ }_{a}\left(\left\langle x^{\prime}\right\rangle_{a}\right)\right) \simeq p_{a}^{b *} \mu_{a}\left(\langle x\rangle_{a},\left\langle x^{\prime}\right\rangle_{a}\right),
$$

since the connecting maps are $H$-maps.

The limit space $H_{F}^{p}(X, H)$ has a continuous multiplication $\mu$ defined as follows:

$$
\mu\left(\langle x\rangle_{a},\langle x\rangle_{b}\right)=\left\langle\mu_{v}\left(p^{v *}{ }_{a}\left(\langle x\rangle_{a}\right), p^{v *}{ }_{b}\left(\langle x\rangle_{b}\right)\right)\right\rangle,
$$

where $\langle x\rangle_{a},\langle x\rangle_{b} \in H_{F}^{p}(X, H)$ and $\alpha, \beta<\nu$.

The definition is independent of the choice of $\nu$ and of the choice of representative of the elements of $H_{F}^{p}(X, H)$ by a standard argument. Denote $\mu\left(\langle x\rangle_{a},\langle x\rangle_{b}\right)$ by $\langle x\rangle_{a} \circ\langle x\rangle_{b}$ and $\langle\theta\rangle_{a}$ by $\theta$ below.

A homotopy-inverse for $H_{F}^{p}(w, \Sigma)$ is $\iota_{w}$, and a homotopy-inverse $\iota$ for $H_{F}^{p}(X, H)$ is defined by $\left(\langle x\rangle_{a}\right)=\left\langle\iota_{a}\left(x_{a}\right)\right\rangle$. The associative and commutative properties of the $H$-structures on the spaces $H_{F}^{p}(w, \Sigma)$ carry over to $H_{F}^{p}(X, H)$ in view of the definition of the $H$-structure on $H_{F}^{p}(X, H)$ above.

If $H$ is a sheaf of $H$-spaces such that $\widetilde{H}$, the sheaf generated by the presheaf of sections of $H$ (see 1.5), is a sheaf of algebraic structures, then the cochain spaces are isomorphic as $H$-spaces. Thus the (Cech) cohomology group with coefficients

(2) Connecting maps satisfy the condition: $p^{v *}{ }^{v p^{w *}}{ }_{v} \simeq p^{w *}{ }_{u}$ (as $H$-maps in this case) whenever $\mu<\nu<\omega[6]$.

(3) Homotopy replaces equality in the usual equivalence relation [5], [6]; this is not the homotopy-limit of Milnor: Morse theory, Princeton Univ. Press, 1963. 
in $\widetilde{H}$ is isomorphic with the cohomology "group" with coefficients in $\widetilde{H}$ (considered as $H$-spaces). It is clear that the above constructions include the ordinary Cech theory.

The following definition will be needed below.

Definition 2.13. Let $A \subset X$, and $i: A \rightarrow X$ an inclusion map. It is assumed that $A$ is locally closed in $X$ if $F$ is a paracompactifying family of supports $\left[2\right.$, p. 139]. The inclusion map induces an onto map $i_{v}^{\#}: C_{F}^{p}(v, \Sigma) \rightarrow$ $C_{F \mid A}^{p}(v \mid A, \Sigma)$, where $v \mid A=\left\{v_{i} \mid v_{i} \cap A \neq \varnothing\right\}$, by the scheme

$$
i_{v}^{\#}\left(f^{p}\right)\left(j_{0} \ldots j_{p}\right)=f^{p}\left(i j_{0} \ldots i j_{p}\right),
$$

where $\left(j_{0} \ldots j_{p}\right) \in v \mid A, f^{p} \in C_{F}^{p}(v, \Sigma)$, and $F \mid A=\{B \in F \mid B \subset A\}$.

Define the relative cochain space by $C_{F}^{p}(v, v \mid A, \Sigma)=\operatorname{Ker} i_{v}^{\#}$. Then, since $d^{p}\left(\operatorname{Ker} i_{v}^{\#}\right) \subset \operatorname{Ker} i_{v}^{\#}$, the relative cohomology space $H_{F}^{p}(v, v \mid A, \Sigma)$ is well defined and inherits a multiplication from $C_{v}^{p}(v, \Sigma)$. Also $i_{v}^{\#}$ induces a map $i_{v}^{*}: H_{F}^{p}(v, \Sigma)$ $\rightarrow H_{F \mid A}^{p}(v \mid A, \Sigma)$, defined by $i_{v}^{*}\left(\left\langle f^{p}\right\rangle\right)=\left\langle i_{v}^{\#} f^{p}\right\rangle$, since $i_{v}^{\#} d^{p} \simeq d^{p} i_{v}^{\#}$.

If $p^{\prime w *}{ }_{v}=p^{w *}{ }_{v} \mid \operatorname{Ker} i_{v}^{*}$, then $\left\{H_{F}^{p}(v, v \mid A, \Sigma), p^{\prime w^{*}}{ }_{v}\right\}$ forms a homotopydirect system [5], [6] and the relative cohomology space is defined as the limit of this system:

$$
H_{F}^{p}(X, A, H)=\underset{w \rightarrow}{L}\left\{H_{F}^{p}(v, v \mid A, \Sigma), p^{\prime w *}\right\}
$$

3. Axioms for sheaf cohomology theory. The above cohomology theory satisfies Cartan's axioms [3] for a sheaf cohomology theory in the setting for sheaves of $H$-spaces. Such a theory is made clear by the following definition.

DEFINITION 3.1. A cohomology theory with coefficients in a sheaf of $\mathrm{H}$ spaces is a covariant $\delta$-functor [7, p. 40] from the category of sheaves of $H$-spaces on a given space to the category of $H$-spaces and $H$-maps which satisfies the following axioms:

I. $H_{F}^{0}(X, H)$ contains a copy of $S_{F}(X, H)$ as a sub- $H$-space.

II. If $0 \rightarrow H^{\prime} \stackrel{\alpha}{\rightarrow} H \stackrel{\beta}{\rightarrow} H^{\prime \prime} \rightarrow 0$ is an exact sequence of sheaves of $H$ spaces on $X$ (see 3.2 below), then the sequence

$$
\cdots \rightarrow H_{F}^{p}\left(X, H^{\prime \prime}\right) \stackrel{\delta^{*}}{\longrightarrow} H_{F}^{p+1}\left(X, H^{\prime}\right) \stackrel{\alpha^{*}}{\longrightarrow} H_{F}^{p+1}(X, H) \stackrel{\beta^{*}}{\longrightarrow} \cdots
$$

is exact (i.e. $\operatorname{Im} \delta^{*} \simeq \operatorname{Ker} \alpha^{*}$ ).

III. $H_{F}^{p}(X, H)=0$ if $H$ is a fine sheaf (see Definition 3.2 below) and $p>0$.

DEFINITION 3.2. A sequence of sheaves of $H$-spaces is exact iff the image of one map is isomorphic with the kernel of the map which follows in the sequence (recall 1.3), that is, the sequence $\cdots \rightarrow H_{i-1} \stackrel{\alpha_{i-1}}{\longrightarrow} H_{i} \stackrel{\alpha_{i}}{\longrightarrow} H_{i+1} \rightarrow$ $\cdots$ is exact iff $\operatorname{Im} \alpha_{i-1} \approx \operatorname{Ker} \alpha_{i}$, where $\operatorname{Ker} \alpha_{i}=\left\{\mathbf{a} \in H_{i} \mid \alpha_{i}(\mathbf{a}) \simeq \theta\right\}$, and 
$\operatorname{Im} \alpha_{i-1}=\left\{\mathbf{a} \in H_{i} \mid \mathbf{a} \simeq \alpha_{i-1}(\mathbf{b})\right\}\left(\alpha_{i}\right.$ as defined in 1.3). An exact sequence of $H$-spaces is defined similarly.

A sheaf of $H$-spaces $H$ is fine iff for each fine covering of $X$ ( $X$ locally compact [2, p. 141]) or locally finite cover of $X,\left\{v_{i}\right\},[7$, p. 74], there exist sheaf maps $\alpha_{i}: H \rightarrow H(1.3)$ such that

$$
\begin{aligned}
& \left|\alpha_{i}\right| \subset \bar{v}_{i}, \\
& \bigcirc \alpha_{i} \simeq 1_{H} .
\end{aligned}
$$

It is clear if $1: H \rightarrow H$ is the identity map, then $1^{*}: H_{F}^{p}(X, H) \rightarrow H_{F}^{p}(X, H)$ is an $H$-isomorphism. Also, if $H^{\prime} \stackrel{\alpha}{\rightarrow} H \stackrel{\beta}{\rightarrow} H^{\prime \prime}$ then $\beta^{*} \alpha^{*}=(\beta \alpha)^{*}: H_{F}^{p}\left(X, H^{\prime}\right) \rightarrow$ $H_{F}^{p}\left(X, H^{\prime \prime}\right)$.

In contrast to the usual situation the following relation holds.

THEOREM 3.3. $H_{F}^{0}(X, H) \supset S_{F}(X, H)$ as a sub-H-space.

Proof. It is assumed $H_{F}^{p}(X, H)$ is trivial for $p<0$, therefore $H_{F}^{0}(v, \Sigma)=$ $\operatorname{Ker} d^{0}$. Clearly every section $s \in S_{F}(X, H)$ determines a cocycle in $H_{F}^{0}(v, \Sigma)$. Thus $S_{F}(X, \dot{H}) \subset H_{F}^{0}(X, \Sigma)(2.1)$.

In order to obtain a stronger relation, additional assumptions must be made on the sheaf space, i.e. the way the stalk structures connect to each other topologically. Thus in the case of sheaves (of algebraic structures) the local maps may be pieced together to obtain a global map or section of $X$ using the discreteness of the stalks.

THEOREM 3.4. Let $0 \rightarrow H^{\prime} \stackrel{\alpha}{\rightarrow} H \stackrel{\beta}{\rightarrow} H^{\prime \prime} \rightarrow 0$ be an exact sequence of sheaves of $H$-spaces on $X$, then there exists a map $\delta^{p}: H_{F}^{p}\left(X, H^{\prime \prime}\right) \rightarrow H_{F}^{p+1}\left(X, H^{\prime}\right)$ such that the sequence

$$
\cdots \rightarrow H_{F}^{p}\left(X, H^{\prime \prime}\right) \stackrel{\delta^{*}}{\longrightarrow} H_{F}^{p+1}\left(X, H^{\prime}\right) \stackrel{\alpha^{*}}{\longrightarrow} H_{F}^{p+1}(X, H) \stackrel{\beta^{*}}{\longrightarrow} \cdots
$$

is exact.

Proof. Let $\nu=\left\{v_{i}\right\}$ be an open cover of $X$ and $N=\bigcap_{m=0}^{q} v_{i_{m}} \neq \varnothing$. Clearly $\alpha^{\#}$ and $\beta^{\#}$ commute with the differentials (2.2).

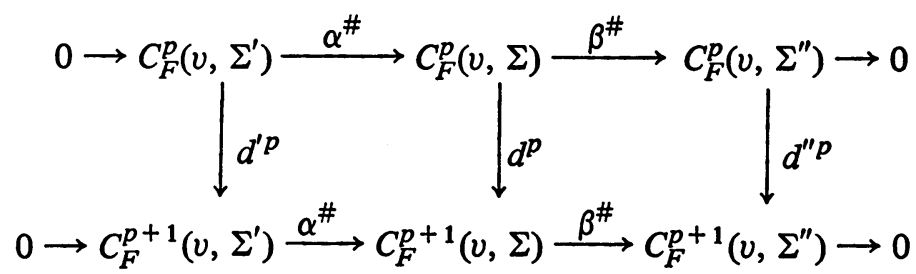

If $\left\langle f^{p}\right\rangle \in H_{F}^{p}\left(v, \Sigma^{\prime \prime}\right)$, define the connecting map $\delta^{p}$ by 


$$
\delta^{p}\left(\left\langle f^{p}\right\rangle\right)=\left\langle\delta^{p} f^{p}\right\rangle=\left\langle\alpha^{\#-1} d^{p} \beta^{\#-1} f^{p}\right\rangle .
$$

That the map $\delta^{p}$ is well defined follows below and $\delta^{p}$ commutes with the connecting maps of the homotopy-direct system.

Let $g_{1}^{p}, g_{2}^{p} \in \beta^{\#-1}\left(f^{p}\right)$, then

$$
d^{p}\left(g_{1}^{p} \circ g_{2}^{p}\right) \simeq d^{p} g_{1}^{p} \circ d^{p} g_{2}^{p} \simeq d^{p} \alpha^{\#} h^{p} \simeq \alpha^{\#} d^{p} h^{p}
$$

for some $h^{p} \in C_{F}^{p}\left(v, \Sigma^{\prime}\right)$, since $g_{1}^{p} \circ l_{2}^{p} \in \operatorname{Ker} \beta^{\#}$. Thus

$$
\left.\left(d^{p} g_{1}^{p} \circ d^{p} g_{2}^{p}\right) \circ\left(\alpha^{\#} d^{p} h^{p}\right) \simeq d^{p} g_{1}^{p} \circ \iota^{p} g_{2}^{p} \circ \alpha^{\#} d^{p} h^{p}\right) \simeq \theta^{p+1},
$$

or $\left\langle\alpha^{\#-1} d^{p} g_{1}^{p}\right\rangle=\left\langle\alpha^{\#-1} d^{p} g_{2}^{p}\right\rangle \in H_{F}^{p+1}(v, \Sigma)$.

The map $\delta^{p}$ is natural by a straightforward argument which is omitted here (see [5]).

A long exact sequence results:

$$
\cdots \rightarrow H_{F}^{p}\left(v, \Sigma^{\prime \prime}\right) \stackrel{\delta^{*}}{\longrightarrow} H_{F}^{p+1}\left(v, \Sigma^{\prime}\right) \stackrel{\alpha^{*}}{\longrightarrow} H_{F}^{p+1}(v, \Sigma) \stackrel{\beta^{*}}{\longrightarrow} \cdots
$$

Exactness at $H_{F}^{p}\left(v, \Sigma^{\prime \prime}\right)$ is demonstrated here with the other two cases following by similar arguments [5].

Let $\left\langle f^{p}\right\rangle \in \operatorname{Im} \beta^{*}$, then $\left\langle f^{p}\right\rangle=\beta^{*}\left(\left\langle g^{p}\right\rangle\right)=\left\langle\beta^{\#} g^{p}\right\rangle$, and

$$
\delta^{*}\left(\left\langle f^{p}\right\rangle\right)=\left\langle\delta^{p} f^{p}\right\rangle=\left\langle\alpha^{\#-1} d^{p} \beta^{\#-1} f^{p}\right\rangle=\left\langle\alpha^{\#-1} d^{p} g^{p}\right\rangle=\left\langle\theta^{p+1}\right\rangle,
$$

or $\operatorname{Im} \beta^{*} \subset \operatorname{Ker} \delta^{*}$.

Let $\left\langle f^{p}\right\rangle \in \operatorname{Ker} \delta^{*}$, then $\delta^{*}\left(\left\langle f^{p}\right\rangle\right)=\left\langle\delta^{p} f^{p}\right\rangle=\left\langle\theta^{p+1}\right\rangle$. But $\delta^{p} f^{p}=$ $\alpha^{\#-1} d^{p} \beta^{\#-1} f^{p}$, so $g^{p} \in \beta^{\#-1} f^{p}$ has the property that $\beta^{*}\left(\left\langle g^{p}\right\rangle\right)=\left\langle f^{p}\right\rangle$.

By passing to the limit of the exact sequences (3.4b) one obtains the desired exact sequence.

THEOREM 3.5. $H_{F}^{p}(X, H)$ is trivial for $p>0$ if $H$ is fine.

PRoof. Let $\nu=\left\{v_{i}\right\}$ be a fine cover (or locally finite cover) (3.2), $\left\{\alpha_{i}\right\}$ a set of sheaf maps with supports in $\left\{\bar{v}_{i}\right\}$ (3.2a), and $\left\{\alpha_{i}^{\prime}\right\}$ the induced maps on the space of sections (1.3).

Define a map $D: C_{F}^{p}(v, \Sigma) \rightarrow C_{F}^{p-1}(v, \Sigma)$, by

$$
\left(D f^{p}\right)\left(i_{0} \ldots i_{p-1}\right)=\underset{j \in \pi}{\bigcirc} \alpha_{j}^{\prime}\left(f^{p}\left(j i_{0} \ldots j i_{p-1}\right)\right),
$$

where $\pi$ is a finite set. Then if $x \in \bigcap_{m=0}^{p-1} v_{i_{m}}\left\langle v_{j},\left(D f^{p}\right)\left(i_{0} \ldots i_{p-1}\right)(x) \simeq \theta_{x}\right.$.

Note that $\left|\alpha_{j}^{\prime} s\right| \subset|s|$ for all $s \in S(X, H)$, thus $\left|D f^{p}\right| \subset\left|f^{p}\right|$. Combining the maps $D$ and $d$ one obtains

$$
d^{p-1} D f^{p}\left(i_{0} \ldots i_{p}\right)=\stackrel{\bigcirc}{p}_{k=0}^{p}(\iota) r_{N}^{N_{k}}\left(\underset{j \in \pi}{\bigcirc} \alpha_{j}^{\prime} f^{p}\left(j i_{0} \ldots \hat{k} \cdots j i_{p}\right)\right)
$$




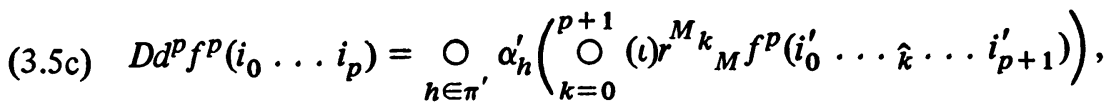
where $i_{0}^{\prime} \ldots i_{p+1}^{\prime}=h i_{0} \ldots h i_{p}$.

In order to obtain nontrivial results $j$ must take on the values $i_{0} \ldots i_{p}$. Combining (3.5b) and (3.5c) one obtains

$$
\left(d^{p-1} D \circ D d^{p}\right) f^{p}\left(i_{0} \ldots i_{p}\right) \simeq \bigcirc_{n=0}^{p} \alpha_{i_{n}}^{\prime} f^{p}\left(i_{0} \ldots i_{p}\right) \simeq f^{p}\left(i_{0} \ldots i_{p}\right) .
$$

Thus $\operatorname{Ker} d^{p} \simeq \operatorname{Im} d^{p-1}$ and $H_{F}^{p}(v, \Sigma)$ is trivial as an $H$-space.

4. Excision. As an application of the above theory an excision theorem is proved (recall 2.13).

THEOREM 4.1. If $U \subset X$ is open, $\bar{U}$ is contained in the interior of $A \subset X$, and $j^{\prime}:(X \backslash U, A \backslash U) \rightarrow(X, A)$ is the inclusion map, then for any family of supports $F$

$$
j^{*}: H_{F}^{*}(X, A, H) \stackrel{\approx}{\longrightarrow} H_{F}^{*}(X \backslash U, A \backslash U, H) .
$$

Proof. Let $\nu$ be an open cover of $X$ and $\omega=j^{-1}(\nu)$ an open cover of $X \backslash U$. Let $v$ and $w$ be the corresponding nerves of the covers $\nu$ and $\omega$. The covers of $X$ are assumed to satisfy: $v_{k} \cap U \neq \varnothing$ implies $v_{k} \subset A$. Such a collection of covers is cofinal in the collection of all open covers of $(X, A)[11$, p. 243].

The following diagram is determined:

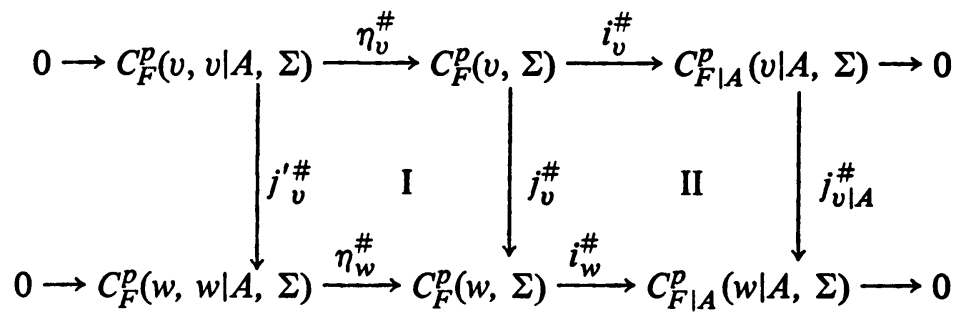

The rows are exact by 2.13 , square I commutes and square II commutes up-to-homotopy. The maps $j_{v}^{\#}$ and $j_{v \mid A}^{\#}$ are isomorphisms since $j\left(i_{0} \ldots i_{p}\right)=$ $\left(i_{0} \ldots i_{p}\right)$ for all $\left(i_{0} \ldots i_{p}\right) \in w(p)$.

The map $j_{v}^{\prime \#}$ is onto, since $\eta_{w}^{\#} j_{v}^{\prime \#}=j_{v}^{\#} \eta_{v}^{\#}$, and satisfies

$$
C_{F}^{p}(v, v \mid A, \Sigma) \approx j_{v}^{\#} \eta_{v}^{\#} C_{F}^{p}(v, v \mid A, \Sigma)=\eta_{w}^{\#} j_{v}^{\#} C_{F}^{p}(v, v \mid A, \Sigma) \approx j_{v}^{\prime \#} C_{F}^{p}(v, v \mid A, \Sigma) .
$$

Thus $j_{v}^{\prime *}$ is an isomorphism and $j^{\prime *}$ is an $H$-isomorphism. 


\section{Homotopy.}

Definition 5.1. If $f: X \rightarrow Y$ and $H$ is a sheaf of $H$-spaces on $Y$, then the inverse image sheaf on $X, f^{*} H$, is defined as the subset of $X \times H$ which satisfies the matching condition $f(x)=p(a)$, where $p: H \rightarrow Y$ is the projection map. Note that $\left(f^{*} H\right)_{x} \approx H_{f(x)}$ as $H$-spaces. An $f$-cohomorphism $f^{*}: H \rightarrow f^{*} H$ is determined by this stalk isomorphism.

DEFinition 5.2. If $f: X \rightarrow Y$ and $H$ is a sheaf of $H$-spaces on $Y$, then $f$ induces a map

$$
f_{w}^{\#}: C^{q}(w, \Sigma) \rightarrow C^{q}\left(f^{-1}(w), \Sigma^{\prime}\right)
$$

by the rule $f_{w}^{\#}\left(g^{q}\right)\left(i_{0} \ldots i_{q}\right)=g^{q}\left(i_{0} \ldots i_{q}\right)$, where $g^{q} \in C^{q}(w, \Sigma),\left(i_{0} \ldots i_{q}\right)$ $\in f^{-1}(w)$ (and thus $\left.\left(i_{0} \ldots i_{q}\right) \in w\right)$, and $\Sigma^{\prime}=\left\{S\left(U, f^{*} H\right), r_{V}^{U}\right\}$. It is clear that $f_{w}^{\#}$ is an $H$-homomorphism, $\left|f_{w}^{\#}\left(g^{q}\right)\right|=\left|g^{q}\right|$ (see 2.1 ), and it may be easily shown that $d f_{w}^{\#} \approx f_{w}^{\#}$.

Define an $H$-homomorphism

$$
f_{w}^{*}: H^{q}(w, \Sigma) \rightarrow H^{q}\left(f^{-1}(w), \Sigma^{\prime}\right)
$$

by $f_{w}^{*}\left(\left\langle g^{q}\right\rangle\right)=\left\langle f_{w}^{\#} g^{q}\right\rangle$.

Let $z=f^{-1}(w), u=f^{-1}(v)$, and $p^{w *}: H^{q}(v, \Sigma) \rightarrow H^{q}(w, \Sigma)$ and $p^{2 *}{ }_{u}: H^{q}\left(u, \Sigma^{\prime}\right) \rightarrow H^{q}\left(z, \Sigma^{\prime}\right)$ be the maps defined in 2.7. Then $f_{w}^{*} p^{w *}{ }_{v} \approx$ $p^{z *}{ }_{u} f_{v}^{*}$, since $f_{w}^{\#} p^{w \#}{ }_{v} \approx p^{z \#}{ }_{u}^{\#}$. Thus $f$ induces a map

$$
f^{*}: H^{q}(Y, H) \rightarrow H^{q}\left(X, f^{*} H\right)
$$

defined as the homotopy-direct limit of the system of maps $\left\{f_{w}^{*}\right\}$. Clearly if $f=1: X \rightarrow X$ then $f^{*}$ is the identity homomorphism, and if $X \stackrel{f}{\rightarrow} Y \stackrel{g}{\rightarrow} Z$, then $(g f)^{*}=f^{*} g^{*}($ cf. 3.2).

Let $q: X \times I \rightarrow X$ be the projection on the first element, and if $t \in I$ let $i_{t}: X \rightarrow X \times I$ be the inclusion defined by $i_{t}(x)=(x, t)$.

THEOREM 5.3. If $H$ is a sheaf of $H$-spaces on the space $X$ and $q$ and $i_{t}$ are the maps defined above, then $i_{t}^{*}: H_{F \times I}^{*}(X \times I, H \times I) \rightarrow H_{F}^{*}(X, H)$ is an H-isomorphism and is independent of $t \in I$.

Proof. Note that $q^{*} H=H \times I$ in view of 5.1. If $\omega$ is an open cover of $X$, then $\omega \times I=q^{-1}(\omega)$ is a cover of $X \times I$ and $q$ induces the map $q_{w}^{\#}: C_{F}^{q}(w, \Sigma)$ $\rightarrow C_{F \times I}^{q}\left(w \times I, \Sigma^{\prime}\right)$, where $\Sigma^{\prime}=\left\{S\left(U, q^{*} H\right), r_{V}^{U}\right\}$. This induced map is an $H$ isomorphism in view of 5.2. Thus $q^{*}$ is an $H$-isomorphism, and since $q i_{t}=1$, $i_{t}^{*} q^{*}=\left(q i_{t}\right)^{*}=1^{*}$, and the map $i_{t}^{*}$ is an $H$-isomorphism independent of $t \in I$. 


\section{BIBLIOGRAPHY}

1. J. F. Adams, H-spaces with few cells, Topology 1 (1962), 67-72. MR 26 \#5574. \#3415.

2. D. G. Bourgin, Modern algebraic topology, Macmillan, New York, 1963. MR 28

3. H. Cartan, Cohomologie des groupes, suites spectral, faisceaux, Séminaire de topologie algébrique de l'Ecole Normal Supérieure, 1950/51. MR 14, 670.

4. R. Godement, Topologie algébrique et théorie des faisceaux, Actualités Sci. Indust., no. 1252, Publ. Math. Univ. Strasbourg, no. 13, Hermann, Paris, 1958. MR 21 \#1583.

5. J. M. Parks, Homotopy-systems, H-spaces and sheaf cohomology, Ph.D. Dissertation, University of Houston, Houston, Tex., 1971.

6. - Applications of homotopy in sheaf theory, Proc. Amer. Math. Soc. 34 (1972), 601-604.

7. R. G. Swan, The theory of sheaves, Univ. of Chicago Press, Chicago, Ill., 1964.

8. A. Dold and R. Thom, Quasifaserungun und unendliche symmetrische Produkte, Ann. of Math. (2) 67 (1958), 239-281. MR 20 \#3542.

9. J. Stasheff, $A$ classification theorem for fibre spaces, Topology 2 (1963), 239246. MR $27 \# 4235$.

10. - H-spaces from a homotopy point of view, Lecture Notes in Math., vol. 161, Springer-Verlag, Berlin and New York, 1970. MR 42 \#5261.

11. S. Eilenberg and N. Steenrod, Foundations of algebraic topology, Princeton Univ. Press, Princeton, N.J., 1952. MR 14, 398.

DEPARTMENT OF MATHEMATICS, UNIVERSITY OF HOUSTON, HOUSTON, TEXAS 77004

DEPARTMENT OF MATHEMATICS, HOUSTON COMMUNITY COLLEGE, HOUSTON, TEXAS 77027

Current address: Department of Mathematics, Howard University, Washington, D. C. 20001 